\title{
Transcriptome Sequencing Reveals the Potential Mechanisms of Modified Electroconvulsive Therapy in Schizophrenia
}

\author{
Wanhong Peng ${ }^{1}$, Qingyu Tan1, Minglan Yu², Ping Wang ${ }^{1}$, Tingting Wang ${ }^{1,3}$, Jixiang Yuan ${ }^{1}$, Dongmei Liư ${ }^{4}$, \\ Dechao Chen ${ }^{4}$, Chaohua Huang1, Youguo Tan ${ }^{5}$, Kezhi Liu1, Bo Xiang ${ }^{1,3}$, and Xuemei Liang ${ }^{1 凶}$ \\ ${ }^{1}$ Department of Psychiatry, Nuclear Medicine and Molecular Imaging Key Laboratory of Sichuan Province, \\ Affiliated Hospital of Southwest Medical University, Luzhou, China \\ ${ }^{2}$ Medical Laboratory Center, Affiliated Hospital of Southwest Medical University, Luzhou, China \\ ${ }^{3}$ Zigong Mental Health Research Center, Zigong Mental Health Center, Zigong, China \\ ${ }^{4}$ Department of Psychiatry, Yibin Fourth People's Hospital, Yibin, China \\ ${ }^{5}$ Zigong Mental Health Center, Zigong, China
}

Objective Schizophrenia (SCZ) is one of the most common and severe mental disorders. Modified electroconvulsive therapy (MECT) is the most effective therapy for all kinds of SCZ, and the underlying molecular mechanism remains unclear. This study is aim to detect the molecule mechanism by constructing the transcriptome dataset from SCZ patients treated with MECT and health controls (HCs).

Methods Transcriptome sequencing was performed on blood samples of 8 SCZ (BECT: before MECT; AECT: after MECT) and 8 HCs, weighted gene co-expression network analysis (WGCNA) was used to cluster the different expression genes, enrichment and protein-protein interaction (PPI) enrichment analysis were used to detect the related pathways.

Results Three gene modules (black, blue and turquoise) were significantly associated with MECT, enrichment analysis found that the long-term potentiation pathway was associated with MECT. PPI enrichment p-value of black, blue, turquoise module are $0.00127,<1 \times 10^{-16}$ and $1.09 \times 10^{-13}$, respectively. At the same time, EP300 is a key node in the PPI for genes in black module, which got from the transcriptome sequencing data.

Conclusion It is suggested that the long-term potentiation pathways were associated with biological mechanism of MECT.

Psychiatry Investig 2021;18(5):385-391

Key Words Electroconvulsive therapy, WGCNA, Network analysis, Schizophrenia.

\section{INTRODUCTION}

Schizophrenia (SCZ) is a stressful, chronic, incorrigible psychological disorder, ${ }^{1}$ which affecting about $1 \%$ of the general population worldwide. ${ }^{2}$ Antipsychotics are the mainstay of treatment, ${ }^{3}$ but it is associated with important side-effects that

\footnotetext{
Received: November 24, 2020 Revised: December 31, 2020 Accepted: March 13, 2021

$\triangle$ Correspondence: Bo Xiang, MD, PhD

Department of Psychiatry, Nuclear Medicine and Molecular Imaging Key Laboratory of Sichuan Province, Affiliated Hospital of Southwest Medical University, No. 25 Taiping Street, Jiangyang District, Luzhou, China

Tel: +86-0830-3165019, Fax: +86-0830-3165019, E-mail: xiangbo@swmu.edu.cn

$\triangle$ Correspondence: Xuemei Liang, MD, $\mathrm{PhD}$

Department of Psychiatry, Nuclear Medicine and Molecular Imaging Key Laboratory of Sichuan Province, Affiliated Hospital of Southwest Medical University, No. 25 Taiping Street, Jiangyang District, Luzhou, China

Tel: +86-0830-3165019, Fax: +86-0830-3165019, E-mail: xuemeiliang@163.com

(c) This is an Open Access article distributed under the terms of the Creative Commons Attribution Non-Commercial License (https://creativecommons.org/licenses/bync/4.0) which permits unrestricted non-commercial use, distribution, and reproduction in any medium, provided the original work is properly cited.
}

can cause serious disability or death. ${ }^{4}$ Although multiple treatments are available for SCZ, antipsychotics remain the primary choice. ${ }^{3}$ But it was reported that compliance with antipsychotic treatment is affected by many factors. ${ }^{4}$

The effectiveness of electroconvulsive therapy (ECT) in SCZ and mood disorders have been verified. ${ }^{5}$ When rapid relief of symptoms is desired, ECT, combined with antipsychotic drugs may be considered an option for patients with SCZ. ${ }^{6}$ In contrast to the high response rate of patients with SCZ, little is known about the underlying mechanisms of the action of ECT.

To uncover the mechanisms of the action of ECT, several studies focus on the change of gene expression. Kaneko et al. found that tumor necrosis factor family may play a role in modified electroconvulsive therapy (MECT) by employing microarray analysis of cDNA derived from the peripheral blood of patients with catatonic SCZ. The expression level of cell factors of patients with SCZ changed after MECT was 
also reported. ${ }^{8}$ Our study aims to detect the possible genes affected by MECT through analyzing the transcriptome of 8 SCZ (before and after MECT) and 8 HCs.

\section{METHODS}

\section{Participants}

All the 8 SCZ were recruited in the Fourth People's Hospital of Yibin, PR China. The Diagnostic and Statistical Manual of Mental Disorders-Fourth Edition (DSM-IV), Patient Edition (SCID-P) ${ }^{9}$ were used to diagnose by the professional trained psychiatrist. All recruited patients meet the enrollment criteria: 1) Age from 18 to 60 years old; 2) The severity of symptom were accessed by the Positive and Negative Syndrome Scale (PANSS) ${ }^{10}$ and the total scores of baseline was 60 or more; 3) MECT were not applied during the last 6 months. The exclusion criteria: The severity physical illnesses including neurologic abnormalities, brain injuries and other related diseases to induce mental symptoms, other mental disorder like dementia and fail to understand the content. The 8 HCs were all recruited from the local area by advertisement and accepted the SCID Non-Patient Edition (SCID-NP) ${ }^{9}$ to affirm the absence of any mental disorders. All the participants were Han Chinese from the Sichuan province of China. The study was approved by the local Institutional Ethics Committee (IRB number: KY201990). All subjects provided informed consent for participation in the study.

\section{MECT treatment}

MECT was applied to all the patients by using the Thymatron IV instrument (Somatics, Lake Bluff, IL, USA). The treatment course of MECT was 6 times, 3 times a week. The patients were evoked using bilateral electrical stimulation with an initial electrical dose that based on $2 / 3$ of their age, and subsequent dosing was performed according to seizure morphology adequacy. EEG can be used to monitor and assess the patient's seizures. The indicators for reference include the peak heart rate, EEG endpoint, average seizure energy index and etc., all the parameters are recommended by instruction of Thymatron. Before treatment, patients received etomidate $\left(0.16^{-}-0.2 \mathrm{mg} / \mathrm{kg}\right)$ to reach anesthesia status, succinylcholine $(1.0 \mathrm{mg} / \mathrm{kg})$ for muscle-relaxing and atropine sulfate $(0.01$ $\mathrm{mL} / \mathrm{kg}$ ) to reduce airway secretion by intravenous injection. The entire treatment process is monitored by professional anesthesiologists to prevent serious side effects such as asphyxia and arrhythmia.

\section{Symptom assessment}

All the patients received two times PANSS (baseline and end of the MECT treatment) to envaulted the severity of symp- toms and the efficiency of the MECT, the PANSS reduction rate was defined as (PANSS score at baseline-PANSS score at the end of ECT treatment)/(PANSS score at baseline-30) $\times 100 \%$, the reduction rate was indicated as: $>75 \%$ indicated complete remission, $50-75 \%$ significant improvement, ${ }^{11}$ so response to ECT was defined as $\geq 50 \%$ PANSS reduction rate and all the patients meet the criterion.

\section{RNA extraction, library preparation, and sequencing}

The peripheral blood of the SCZ patients were collected before and after the MECT in anticoagulation tubes and stored in $-80^{\circ} \mathrm{C}$ immediately. The whole blood of the HCs were collected in the same way. All the whole blood samples were used to extract the RNA using the protocol for TRIzol Reagent. The NanoDrop 2000 spectrophotometer (NanoDrop Technologies, Wilmington, Delaware) and Agilent 2100 (Agilent Technologies, Santa Clara, CA, USA) were used respectively to determine the RNA concentration and integrity number (IN), besides, agarose gel electrophoresis proved the integrity of all the RNA samples, spectrophotometer shows the OD260/ 280 of all the samples is between 1.8 to 2.0. To construct the RNA-transcriptome library, 5 ug of each high-quality RNA sample was used. The libraries were used for further transcriptome sequencing. In brief, mRNA was isolated according to the poly(A)-oligo(dT) and fragmented by fragmentation buffer, secondly, cDNA was synthesized via random hexamers and Illumina's library construction protocol was used to handle matched cDNA. Libraries were size-selected for cDNA target fragments of 200-300 bp on 2\% Low Range Ultra Agarose followed by PCR amplified using Phusion DNA polymerase for 15 PCR cycles. After quantification by TBS-380, a pairedend RNA-seq sequencing library was sequenced with the Illumina HiSeq 4000 system (2×150 bp read length).

\section{Read mapping and the construction of the library}

SeqPrep (https://github.com/jstjohn/SeqPrep) and Sickle (https://github.com/najoshi/sickle) were used to test the quality of raw paired-end reads and detect the default parameters. Then using TopHat (http://tophat.cbcb.umd.edu/, version 2.0.0) 12 software to respectively aligned the clean reads in accordance with the reference genome. The criteria of bowtie mapping were as follows: sequencing reads should be particular matched to the genome with 2 mismatches at most and no insertions or deletions. Under the principle of the whole genome was split into multiple $15 \mathrm{kbp}$ windows that shared $5 \mathrm{kbp}$. New transcribed regions were defined as more than 2 consecutive windows without overlapped gene regions, where at least 2 reads mapped per window in the same orientation. To identify DEGs between BECT and AECT patients. Expression level of each gene was calculated using the frag- 
ments per kilobase of exon per million mapped reads (FRKM) method. RNA Sequencing by Expectation-Maximization (RSEM) (http://deweylab.biostat.wisc.edu/rsem/) was used to define gene abundances.

\section{Construct the WGCNA network}

The $\mathrm{R}$ package software edge $\mathrm{R}^{12}$ was used for differential expression analysis, then the R package WGCNA ${ }^{13}$ was used to construct gene co-expression networks based on the different expression genes (DEGs), and the minimum module size was set to 30. Enrichment and PPI analysis were in the supplementary material.

\section{Enrichment analysis}

To determine whether the genes in each module were associated with pathophysiological mechanism of SCZ, Kyoto Encyclopedia of Genes and Genomes (KEGG) and Disease Ontology (DO) enrichment analysis of those genes were applied. A hypergeometric test implemented in WebGestalt ${ }^{14}$ allowed us to compute the enrichment p-value, which was followed by a Benjamini-Hochberg $(\mathrm{BH})$ correction for multiple testing. The enriched results were reported at a $\mathrm{BH}$-corrected value of $\mathrm{p}<0.05$.

\section{Protein-protein interaction analysis}

We carried out the permutation test using STRING ${ }^{15}$ and evaluated whether genes in the three gene module (Blue, Black and Turquise) had significant physical interactions with each other or with other proteins via the network connectivity parameters (number of edges and degree) versus the random networks with a similar size and degree distribution. Then we utilized the CentiScaPe $2.2^{16}$ tool to identify the key node according to the topological properties of the degree and betweenness in black module.

\section{Gene expression of EP300 in between SCZ and HCs in different brain areas}

In order to know whether the expression level of EP300 in different human brain areas have discrepancy, by examining 5 brain regions (cerebellum, parietal cortex brain, hippocampus, prefrontal cortex and striatum) gene expression data of SCZ patients and HCs (all data from http://www.szdb.org/ index.html).

\section{Statistical analyses}

Multiple analysis was used to test those modules in different group and each module needs to meet the following points: 1) statistical differences for MEs between BECT and HC; 2) statistical differences for MEs between BECT and AECT group; 3) no statistical difference for MEs between AECT and HC group. Chi-square test were used to examine the differences of age, gender, marital status resident areas; t-test was used to examine the differences of educated years of patients and HCs, and PANSS scores (BECT and AECT) also use t-test to check the statistic difference. SPSS version 23 (IBM Corp., Armonk, NY, USA) was used to all the data analysis.

\section{RESULTS}

\section{Description of clinical information}

The demographic differences and statistic results are all shown in the Table 1. There is no statistical variability between SCZ and $\mathrm{HC}$ group in age, gender, educated years, marriage status and urban-rural differences. Among the 8 SCZ patients, before the MECT they all received the antipsychotic medication treatment, in addition to the use of antipsychotic drugs during the MECT, 2 patients used propranolol to control their heart rate, 2 patients used a combination of benzodiazepines to improve sleep quality, and another patient add a mood stabilizer; except one female patient has the positive family history of mental disorder, others are all negative family history. PANSS scores and other clinical information are shown in Table 1.

\section{Construct the WGCNA network}

To investigate the expression of genes affected by MECT in SCZ. Firstly, 3000 DEGs $(p<0.05)$ were obtained after analyzing the transcriptome sequence of 8 SCZ patients, then those DEGs of the 8 SCZ patients and $8 \mathrm{HCs}$ were used to construct the gene co-expression network through WGCNA and 13 modules were obtained.

\section{Compare the MEs of each module}

According to the above-mentioned WGCNA analysis, multiple comparisons were used to compare MEs in each module in SCZ patients (BECT vs. AECT) and HCs, it found that three modules (Black, Blue, and Turquoise) whose MEs were significantly associated with disease and treatment status (Table 2).

\section{Gene sets enrichment analysis}

Results of KEGG pathway are following points: One important pathway in black module was found: long-term potentiation (Table 3, Supplementary Table 1 in the online-only Data Supplement). Other pathways also have the close connections to mental, nervous disorders, chromosome aberrations and so on (Supplementary Table 2 in the online-only Data Supplement). Besides, it was found that the black module was also associated with drug valproic acid (Table 3, Supplementary Table 3 in the online-only Data Supplement). 
Table 1. Demographic and clinical data

\begin{tabular}{|c|c|c|c|c|}
\hline Variables & $\mathrm{SCZ}(\mathrm{N}=8)$ & $\mathrm{HC}(\mathrm{N}=8)$ & $\chi^{2} / t$ & $\mathrm{p}$ \\
\hline Age, mean & $31.63 \pm 6.28$ & $31.25 \pm 11.22$ & $\mathrm{t}=0.30$ & 0.75 \\
\hline Gender, women (\%) & $4(50)$ & $4(50)$ & $\chi^{2}=0$ & 1 \\
\hline \multicolumn{5}{|l|}{ Educated years, N (\%) } \\
\hline Primary & $0(0)$ & $2(25)$ & $\chi^{2}=3.54$ & 0.17 \\
\hline Middle & $5(62.5)$ & $3(37.5)$ & $\chi^{2}=2.4$ & 0.30 \\
\hline High \& above & $3(37.5)$ & $3(37.5)$ & $\chi^{2}=0$ & 1.00 \\
\hline Baseline PANSS score & $83.63 \pm 7.11$ & & $\mathrm{t}=1.36$ & 0.19 \\
\hline AECT PANSS score & $39.88 \pm 6.92$ & & $t=6.07$ & $<0.01$ \\
\hline \multicolumn{5}{|l|}{ Medicine, N (\%) } \\
\hline Risperidone (3-6 mg/d) & $4(50)$ & - & & \\
\hline Sulpiride $(0.3-0.6 \mathrm{~g} / \mathrm{d})$ & $2(25)$ & - & & \\
\hline Aripiprazole (10-15 mg/d) & $2(25)$ & - & & \\
\hline Married (\%) & $4(50)$ & $4(50)$ & & \\
\hline Urban \& rural (\%) & $6(75)$ & $6(75)$ & & \\
\hline
\end{tabular}

Statistic demographic data results, there is three levels in educated years, primary refer to the 1-6 educated years, middle refer to 7-12 years and high \& above refer to 13 or longer educated years. SCZ: schizophrenia, HCs: health controls, BECT: before MECT, AECT: after MECT, PANSS: Positive and Negative Syndrome Scale

\section{PPI network analysis of genes in the three gene modules}

The findings indicated that the richness in protein connections in each module and the resulting network was significantly different from any random networks; there were 51 direct edges in the black module gene network compared with only 32 edges expected ( $\mathrm{p}=0.00127)$ (Supplementary Figure $1 \mathrm{~A}$ in the online-only Data Supplement), 493 direct edges in the blue module gene network compared with only 309 edges expected $(\mathrm{p}<0.001)$ (Supplementary Figure 1B in the onlineonly Data Supplement), 653 direct edges in the turquoise module network compared with only 483 edges expected $(\mathrm{p}<0.001)$ (Supplementary Figure 1C in the online-only Data Supplement). Those results suggest that the networks generated from genes in the three gene modules did not occur by chance. Meanwhile, EP300 is also a core node in the PPI network for black module, which has 10 degrees and 17.3 betweenness (Supplementary Table 4 in the online-only Data Supplement).

\section{Expression of key gene EP300 in different brain areas between SCZ and HCs}

By examining the gene expression level of EP300 in the brain regions mentioned above, we identified three areas including hippocampus $(\mathrm{p}=0.024)$, prefrontal cortex $(\mathrm{p}=0.012)$, cerebellum $\left(\mathrm{p}=1.35 \times 10^{-4}\right)$. After the false discovery rate (FDR), it found that gene expression level of EP300 in cerebellum was statistic different $(\mathrm{FDR}=0.026)$ (Figure 1). Besides, from the results of hub genes in PPI for black module, it found that EP300 was hub gene, which participated in LTP pathway
Table 2. Multiple comparisons of 13 module eigengenes

\begin{tabular}{lccc}
\hline \multicolumn{1}{c}{ Module } & BECT \& AECT & BECT \& HCs & AECT \& HCs \\
\hline Tan & 0.001 & 0.277 & 0.010 \\
Black & 0.003 & $<0.001$ & 0.352 \\
Pink & 0.014 & $<0.001$ & 0.045 \\
Blue & 0.006 & 0.009 & 0.827 \\
Turquoise & 0.026 & 0.004 & 0.405 \\
Magenta & 0.087 & 0.159 & 0.742 \\
Salmon & 0.317 & 0.311 & 0.991 \\
Green & 0.539 & 0.547 & 0.991 \\
Yellow & 0.537 & 0.220 & 0.532 \\
Greenyellow & 0.758 & 0.917 & 0.680 \\
Purple & 0.778 & 0.432 & 0.611 \\
Brown & 0.754 & 0.714 & 0.498 \\
Red & 0.959 & 0.867 & 0.908 \\
\hline
\end{tabular}

HCs: health controls, AECT: patients before modified electroconvulsive therapy, BECT: patients after modified electroconvulsive therapy

meanwhile.

\section{DISCUSSION}

It was found that gene expression changes in patients treated with MECT. Nishiguchi et al. ${ }^{17}$ found the mRNA expression of Yamanaka's four transcription factors in the peripheral blood showed a tendency toward higher levels after MECT, the mRNA level of synapsin II was significantly upregulated 
Table 3. Results of enrichment analysis for genes in the black module

\begin{tabular}{clcccc}
\hline \multicolumn{1}{c}{ Category } & \multicolumn{1}{c}{ Pathway } & ID & P & P adjust & Gene module \\
\hline KEGG & Long-term potentiation & 4720 & $5.49 \mathrm{e}-05$ & 0.0016 & Black \\
& Phosphatidylinositol signaling system & 4070 & 0.0004 & 0.0058 & Black \\
& Regulation of actin cytoskeleton & 4810 & 0.0004 & 0.0058 & Black \\
Drug & Valproic acid & PA451846 & 0.0074 & 0.0222 & Black \\
\hline
\end{tabular}
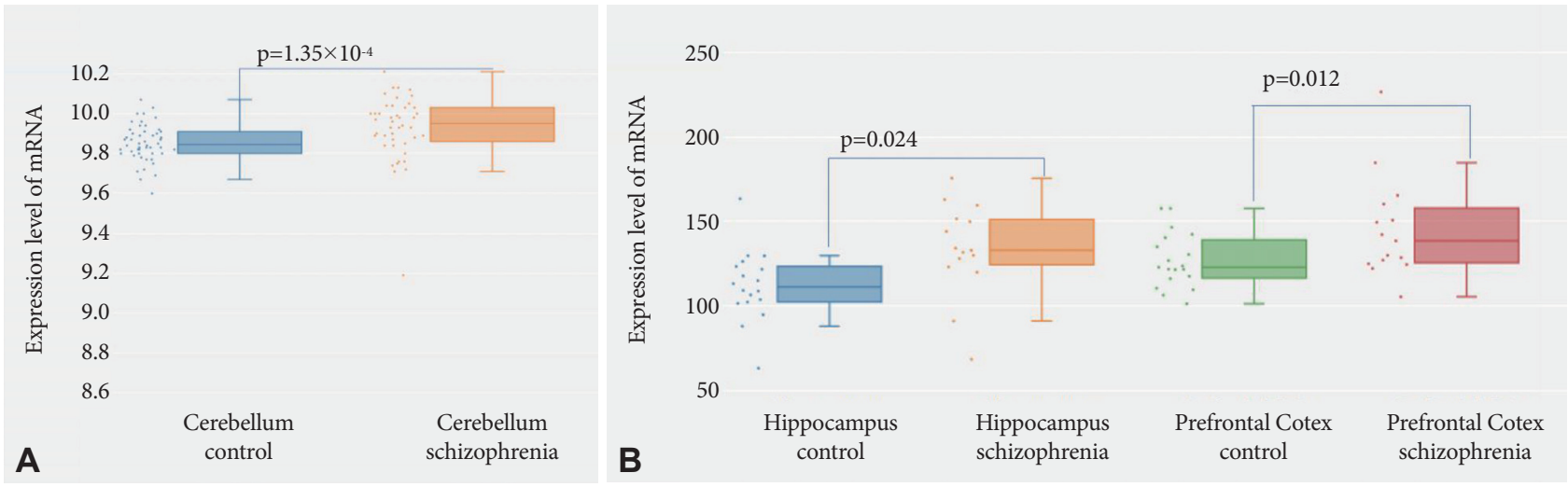

Figure 1. Gene expression level of EP300 in different brain areas. A: The gene expression levels of EP300 in cerebellum areas between patients with SCZ and controls. B: The gene expression levels of EP300 were compared in hippocampus and prefrontal cortex between patients with SCZ and controls. The X-axis indicates the gene expression level of EP300 in different brain regions, and the Y-axis indicates the amount of gene expression.

after repeated ECS. ${ }^{18}$ To our knowledge, this is the first study focus on the differences of transcriptomes between SCZ patients treated with MECT and HCs.

Long-term potentiation (LTP) is also an important pathway in black module, it participated in variety of SCZ-related biological pathways. Like long-term plasticity of synaptic transmission, such as in LTP and long-term depression (LTD), provides a cellular correlate of experience-driven learning. ${ }^{19}$ As identified by GWAS, ${ }^{20}$ shared KEGG pathways related to SCZ including LTP. ${ }^{20}$ However, variation in practice of ECT can lead to cognitive side-effects, ${ }^{21}$ which memory impairment is the most worrisome, it is associated with both anterograde and retrograde amnesia, ${ }^{22}$ although a recently study concluded that there is no evidence of cumulative cognitive deficits associated with repeated ECT courses. ${ }^{23}$ Converging lines of evidence suggest that cognitive deficits are associated with impaired LTP. Alterations of dopaminergic, GABAergic, and glutamatergic neurotransmission could all lead to impaired LTP in SCZ, ${ }^{24}$ both LTP and LTD represent morphological and functional changes occurring in the process of memory formation. ${ }^{25}$ Our results identified this important signal pathways could provide new clue for understanding the genetic architecture of MECT in SCZ.

DO enrichment analysis showed that genes in the black module were related to valproic acid (VPA). VPA was found to induce broader epigenetic changes through different mechanisms, like DNA demethylation and histones acetylation. ${ }^{26}$
Genotypic variations can influence methylation levels of the CHRNA7 promoter, which is linked with SCZ, it has been proven that epigenetic dysregulation can be reversed by valproate, which caused demethylation and increased CHRNA7 expression in Hela cells. ${ }^{27}$ Combine the found of Paulsen et al. ${ }^{28}$ they revealed the presence of elevated levels of potassium and zinc in SCZ NPCs (neural progenitor cells), neural cells treated with valproate brought potassium and zinc content back to control levels. Besides, an animal study focus on plasticity-related mechanisms in the neocortex of 2-week-old rats prenatally exposed to VPA, it indicate that VPA significantly enhances NMDA receptor-mediated transmission and causes increased plasticity in the neocortex. ${ }^{29}$ More importantly, a study shows that treatment with VPA make the enrichment of Ep300 and Kdm6b in PP but not PE of Pou5f1 promoter, which POU5F1 is essential for maintaining pluripotency in embryonic stem cells (ESCs). ${ }^{30}$

In summaries, VPA plays an important role in SCZ and symptoms relief in SCZ may partly rely on MECT mediated VPA pathway alteration.

It has known to all that MECT also could improve the cognitive function. it was indicated that eight genes of some loci were associated with cognitive deficits of SCZ, EP300 is one of it. ${ }^{31}$ In addition, EP300 may become the key gene to influence the epigenetic mechanisms, which including gene expression, and synaptic plasticity. ${ }^{31}$ A study using a combination of relative enrichment score (RES) and conditional FDR 
to elucidate the links between neuroticism and complex diseases, it was found that three SNPs are located in intronic regions of EP300 which have been reported to be associated with autism, Parkinson's disease and SCZ, respectively. ${ }^{32} \mathrm{Be}-$ sides, single SNP analyses revealed that rs9607782, located near EP300, was significantly associated with amygdala recruitment during emotion processing. ${ }^{33}$ A mutation burden test found gene the strawberry notch homolog 1 (SBNO1), in the exons of this gene found different mutation burden profile from the EP300, ${ }^{34}$ which loss of one copy of EP300 leads to abnormal neurodevelopment. ${ }^{35}$ From all those findings, it can confirmed that EP300 is not only the key gene in black module, but also participated in so many functions related to SCZ, it can infer that MECT may change the related pathways, and further studies are needed to reveal the underlying mechanism of effects of EP300 on cognitive function, which may expand our understanding of how MECT improves cognitive impairment in SCZ.

In conclusion, our work still has some shortcomings and limitations. The insufficient amount of the samples is a great shortage; the influence of all the antipsychotics should be considered into the assessment of the severity of the symptoms, the impact of mono-antipsychotic, MECT and antipsychotics combined MECT treatment to gene expressions levels should be considered in the future researches, the gene expression changes in different time points in MECT therapy, the gene expression profile of MECT-resistance patients and etc. What is more, the transcriptome data of other SCZ related brain areas should also be detected to compare the DEGs to find more possible pathways. Besides, the pathways that revealed by our transcriptome data play a vital role in multiple neural functions, include cognitive function, neurodevelopment, synaptic plasticity and etc.

From all the above results, it is informed that many previous studies and findings were based on the change of single gene and/or protein of SCZ. Our study identified the long-term potentiation pathway and gene EP300 may play an important role in MECT and provides a new and macro perspectives for the future studies which devoted to understand the possible mechanism of MECT in SCZ treatment.

\section{Supplementary Materials}

The online-only Data Supplement is available with this article at https://doi.org/10.30773/pi.2020.0410.

\section{Acknowledgments}

This study was supported in part by a grant from National Nature Science Foundation of China (82001414, BX), Ministry of education Chunhui plan [2020 (703)], Sichuan provincial health and Family Planning Commission (16PJ562), Key projects of the Sichuan Provincial Education Department (18ZA0534), Luzhou Science and Technology Bureau [2017-S-40(4/18), 2016-S-67(7/23)], Youth Project of Affiliated Hospital of Southwest Medi- cal University [2017-PT-9, 2011(37)], Southwest Medical University-Luzhou Government [2015LZCYD-S06(5/11), 2017LZXNYD-Z02] and Open Program of Nuclear Medicine and Molecular Imaging Key Laboratory of Sichuan Province.

\section{Conflicts of Interest}

The authors have no potential conflicts of interest to disclose.

\section{Author Contributions}

Conceptualization: Wanhong Peng, Qingyu Tan, Ping Wang, Bo Xiang, Kezhi Liu, Xuemei Liang. Data curation: Wanhong Peng, Qingyu Tan, Minglan Yu, Ping Wang, Tingting Wang, Jixiang Yuan. Formal analysis: Wanhong Peng, Qingyu Tan, Ping Wang, Bo Xiang. Funding acquisition: Wanhong Peng, Dongmei Liu, Dechao Chen, Chaohua Huang, Youguo Tan, Kezhi Liu, Bo Xiang, Xuemei Liang. Investigation: Wanhong Peng, Qingyu Tan, Minglan Yu, Ping Wang, Tingting Wang, Jixiang Yuan. Methodology: Wanhong Peng, Qingyu Tan, Ping Wang, Bo Xiang. Project administration: Wanhong Peng, Dongmei Liu, Dechao Chen, Chaohua Huang, Youguo Tan, Kezhi Liu, Bo Xiang, Xuemei Liang. Resources: Wanhong Peng, Dongmei Liu, Dechao Chen, Kezhi Liu, Bo Xiang, Xuemei Liang. Validation Software: Wanhong Peng, Ping Wang, Bo Xiang. Supervision: Dongmei Liu, Dechao Chen, Chaohua Huang, Youguo Tan, Kezhi Liu, Bo Xiang, Xuemei Liang. Validation: all authors. Visualization: all authors. Writingoriginal draft: Wanhong Peng. Writing—review \& editing: all authors.

\section{ORCID iDs}

Wanhong Peng

Qingyu Tan

Minglan Yu

Ping Wang

Tingting Wang

Jixiang Yuan

Dongmei Liu

Dechao Chen

Chaohua Huang

Youguo Tan

Kezhi Liu

Bo Xiang

Xuemei Liang
https://orcid.org/0000-0002-7785-435X

https://orcid.org/0000-0001-6536-8074 https://orcid.org/0000-0003-2785-6733 https://orcid.org/0000-0001-7095-8669 https://orcid.org/0000-0003-1332-8980 https://orcid.org/0000-0003-1130-1901 https://orcid.org/0000-0001-7905-4615 https://orcid.org/0000-0001-9515-1685 https://orcid.org/0000-0002-3169-3749 https://orcid.org/0000-0001-8700-0502 https://orcid.org/0000-0003-4515-4209 https://orcid.org/0000-0001-8341-2286 https://orcid.org/0000-0002-1894-7660

\section{REFERENCES}

1. Fatima W, Riaz S, Aiman Shahzad M, Naz Z, Mahmood S, Hasnain S. Chromosomal region 1q24. 1 is associated with increased risk of schizophrenia in Pakistani population. Gene 2020;734:144390.

2. Leucht S, Davis J, Engel R, Kissling W, Kane JM. Definitions of response and remission in schizophrenia: recommendations for their use and their presentation. Acta Psychiatr Scand Suppl 2009;(438):7-14.

3. Leucht S, Cipriani A, Spineli L, Mavridis D, Orey D, Richter F, et al. Comparative efficacy and tolerability of 15 antipsychotic drugs in schizophrenia: a multiple-treatments meta-analysis. Lancet 2013;382:951-962.

4. Oehl M, Hummer M, Fleischhacker WW. Compliance with antipsychotic treatment. Acta Psychiatr Scand Suppl 2000;(407):83-86.

5. Tang YL, Jiang W, Ren YP, Ma X, Cotes RO, McDonald WM. Electroconvulsive therapy in China: clinical practice and research on efficacy. J ECT 2012;28:206-212.

6. Tharyan P, Adams CE. Electroconvulsive therapy for schizophrenia. Cochrane Database Syst Rev 2005;(2):CD000076.

7. Kaneko T, Kanazawa T, Nishiguchi M, Kikuyama H, Tsutsumi A, Uenishi $\mathrm{H}$, et al. Microarray analysis of human blood during electroconvulsive therapy. J ECT 2015;31:234-237.

8. Benros ME, Nielsen PR, Nordentoft M, Eaton WW, Dalton SO, Mortensen $\mathrm{PB}$. Autoimmune diseases and severe infections as risk factors for schizophrenia: a 30-year population-based register study. Am J Psychiatry 2011; 
168:1303-1310.

9. First MB. User's Guide for the Structured Clinical Interview for DSMIV Axis II Personality Disorders: SCID-II. Washington, DC: American Psychiatric Press, 1997, p.91.

10. Kay SR, Fiszbein A, Opler LA. The positive and negative syndrome scale (PANSS) for schizophrenia. Schizophr Bull 1987;13:261-276.

11. Guo YF, Fu HB, Liu ZY, Lu W, Luo KY, Zhu HR, et al. Effects of the modified electric convulsive treatment (MECT) on cell factors of schizophrenia. Exp Ther Med 2017;13:873-876.

12. Robinson MD, McCarthy DJ, Smyth GK. edgeR: a Bioconductor package for differential expression analysis of digital gene expression data. Bioinformatics 2010;26:139-140.

13. Langfelder P, Horvath S. WGCNA: an R package for weighted correlation network analysis. BMC Bioinformatics 2008;9:559.

14. Liao Y, Wang J, Jaehnig EJ, Shi Z, Zhang B. WebGestalt 2019: gene set analysis toolkit with revamped UIs and APIs. Nucleic Acids Res 2019; 47:W199-W205

15. Szklarczyk D, Morris JH, Cook H, Kuhn M, Wyder S, Simonovic M, et al. The STRING database in 2017: quality-controlled protein-protein association networks, made broadly accessible. Nucleic Acids Res 2017; 45:D362-D368.

16. Scardoni G, Petterlini M, Laudanna C. Analyzing biological network parameters with CentiScaPe. Bioinformatics 2009;25:2857-2859.

17. Nishiguchi M, Kikuyama H, Kanazawa T, Tsutsumi A, Kaneko T, Uenishi $\mathrm{H}$, et al. Increases in iPS transcription factor (Oct4, Sox2, c-Myc, and Klf4) gene expression after modified electroconvulsive therapy. Psychiatry Investig 2015; 12: 532-537.

18. Gonçalves VF, Cappi C, Hagen CM, Sequeira A, Vawter MP, Derkach A, et al. A comprehensive analysis of nuclear-encoded mitochondrial genes in schizophrenia. Biol Psychiatry 2018;83:780-789.

19. Grasselli G, Hansel C. Cerebellar long-term potentiation: cellular mechanisms and role in learning. Int Rev Neurobiol 2014;117:39-51.

20. Wang Z, Li P, Wu T, Zhu S, Deng L, Cui G. Axon guidance pathway genes are associated with schizophrenia risk. Exp Ther Med 2018;16: 4519-4526.

21. Galvez V, Li A, Oxley C, Waite S, De Felice N, Hadzi-Pavlovic D, et al. Health Related Quality of Life after ECT for depression: a study exploring the role of different electrode-placements and pulse-widths. J Affect Disord 2016;206:268-272.

22. Weiner RD, Reti IM. Key updates in the clinical application of electroconvulsive therapy. Int Rev Psychiatry 2017;29:54-62.

23. Kirov GG, Owen L, Ballard H, Leighton A, Hannigan K, Llewellyn D, et al. Evaluation of cumulative cognitive deficits from electroconvulsive therapy. Br J Psychiatry 2016;208:266-270.
24. Salavati B, Rajji TK, Price R, Sun Y, Graff-Guerrero A, Daskalakis ZJ. Imaging-based neurochemistry in schizophrenia: a systematic review and implications for dysfunctional long-term potentiation. Schizophr Bull 2015;41:44-56.

25. Sibarov DA, Antonov SM. Calcium-Dependent Desensitization of NMDA Receptors. Biochemistry (Mosc) 2018;83:1173-1183.

26. Gardea-Resendez M, Kucuker MU, Blacker CJ, Ho AM, Croarkin PE, Frye MA, et al. Dissecting the epigenetic changes induced by non-antipsychotic mood stabilizers on schizophrenia and affective disorders: a systematic review. Front Pharmacol 2020;11:467.

27. Dyrvig M, Mikkelsen JD, Lichota J. DNA methylation regulates CHRNA7 transcription and can be modulated by valproate. Neurosci Lett 2019;704:145-152.

28. Paulsen Bda S, Cardoso SC, Stelling MP, Cadilhe DV, Rehen SK. Valproate reverts zinc and potassium imbalance in schizophrenia-derived reprogrammed cells. Schizophr Res 2014;154:30-35.

29. Rinaldi T, Kulangara K, Antoniello K, Markram H. Elevated NMDA receptor levels and enhanced postsynaptic long-term potentiation induced by prenatal exposure to valproic acid. Proc Natl Acad Sci U S A 2007;104:13501-13506.

30. Wang WP, Tzeng TY, Wang JY, Lee DC, Lin YH, Wu PC, et al. The EP300, KDM5A, KDM6A and KDM6B chromatin regulators cooperate with KLF4 in the transcriptional activation of POU5F1. PLoS One 2012;7: e52556.

31. Whitton L, Cosgrove D, Clarkson C, Harold D, Kendall K, Richards A, et al. Cognitive analysis of schizophrenia risk genes that function as epigenetic regulators of gene expression. Am J Med Genet B Neuropsychiatr Genet 2016;171:1170-1179.

32. Hadjidaniel MD, Muthugounder S, Hung LT, Sheard MA, Shirinbak S, Chan RY, et al. Tumor-associated macrophages promote neuroblastoma via STAT3 phosphorylation and up-regulation of c-MYC. Oncotarget 2017;8:91516-91529.

33. Erk S, Mohnke S, Ripke S, Lett TA, Veer IM, Wackerhagen C, et al. Functional neuroimaging effects of recently discovered genetic risk loci for schizophrenia and polygenic risk profile in five RDoC subdomains. Transl Psychiatry 2017;7:e997.

34. Girard SL, Dion PA, Bourassa CV, Geoffroy S, Lachance-Touchette P, Barhdadi A, et al. Mutation burden of rare variants in schizophrenia candidate genes. PLoS One 2015;10:e0128988.

35. Roelfsema JH, White SJ, Ariyurek Y, Bartholdi D, Niedrist D, Papadia F, et al. Genetic heterogeneity in Rubinstein-Taybi syndrome: mutations in both the CBP and EP300 genes cause disease. Am J Hum Genet 2005; 76: $572-580$ 
Supplementary Table 1. The results of KEGG enrichment analysis of genes in three gene modules

\begin{tabular}{|c|c|c|c|c|}
\hline KEGG pathway & KEGG ID & $\mathrm{P}$ & $P_{\text {adjust }}$ & Gene module \\
\hline Long-term potentiation & 4720 & $5.49 \mathrm{e}-05$ & 0.0016 & Black \\
\hline Regulation of actin cytoskeleton & 4810 & 0.0004 & 0.0058 & Black \\
\hline Phosphatidylinositol signaling system & 4070 & 0.0016 & 0.0155 & Black \\
\hline Leishmaniasis & 5140 & 0.0189 & 0.0520 & Black \\
\hline Focal adhesion & 4510 & 0.0211 & 0.0520 & Black \\
\hline Hypertrophic cardiomyopathy (HCM) & 5410 & 0.0247 & 0.0520 & Black \\
\hline Calcium signaling pathway & 4020 & 0.0153 & 0.0520 & Black \\
\hline Wnt signaling pathway & 4310 & 0.0098 & 0.0520 & Black \\
\hline Endometrial cancer & 5213 & 0.0102 & 0.0520 & Black \\
\hline Prostate cancer & 5215 & 0.0281 & 0.0520 & Black \\
\hline Osteoclast differentiation & 4380 & $2.18 \mathrm{e}^{-12}$ & $2.57 \mathrm{e}-10$ & Blue \\
\hline Hepatitis C & 5160 & $4.45 \mathrm{e}^{-12}$ & $2.63 e-10$ & Blue \\
\hline Toll-like receptor signaling pathway & 4620 & $1.43 \mathrm{e}^{-11}$ & $5.62 \mathrm{e}-10$ & Blue \\
\hline Neurotrophin signaling pathway & 4722 & $2.87 \mathrm{e}^{-10}$ & $8.47 \mathrm{e}-09$ & Blue \\
\hline Pancreatic cancer & 5212 & $5.00 \mathrm{e}-10$ & $1.13 \mathrm{e}-08$ & Blue \\
\hline Non-small cell lung cancer & 5223 & $5.74 \mathrm{e}^{-10}$ & $1.13 \mathrm{e}-08$ & Blue \\
\hline Leukocyte transendothelial migration & 4670 & $1.01 \mathrm{e}-09$ & $1.70 \mathrm{e}-08$ & Blue \\
\hline Fc epsilon RI signaling pathway & 4664 & $1.90 \mathrm{e}-09$ & $2.80 \mathrm{e}-08$ & Blue \\
\hline $\mathrm{T}$ cell receptor signaling pathway & 4660 & $4.93 \mathrm{e}-09$ & $6.46 \mathrm{e}-08$ & Blue \\
\hline Prostate cancer & 5215 & $6.94 \mathrm{e}-09$ & $8.19 \mathrm{e}-08$ & Blue \\
\hline Protein processing in endoplasmic reticulum & 4141 & $1.51 \mathrm{e}-09$ & $1.83 \mathrm{e}-07$ & Turquoise \\
\hline Ubiquitin mediated proteolysis & 4120 & $3.86 \mathrm{e}-08$ & $1.56 \mathrm{e}-06$ & Turquoise \\
\hline Endocytosis & 4144 & $2.98 \mathrm{e}-08$ & $1.56 \mathrm{e}-06$ & Turquoise \\
\hline Hepatitis C & 5160 & $2.58 \mathrm{e}-07$ & $7.80 \mathrm{e}-06$ & Turquoise \\
\hline Cell cycle & 4110 & $7.62 \mathrm{e}-07$ & $1.84 \mathrm{e}-05$ & Turquoise \\
\hline Neurotrophin signaling pathway & 4722 & $9.86 \mathrm{e}-07$ & $1.99 \mathrm{e}-05$ & Turquoise \\
\hline Prostate cancer & 5215 & $1.62 \mathrm{e}-06$ & $2.31 \mathrm{e}-05$ & Turquoise \\
\hline Pathways in cancer & 5200 & $1.58 \mathrm{e}-06$ & $2.31 \mathrm{e}-05$ & Turquoise \\
\hline Renal cell carcinoma & 5211 & $1.72 \mathrm{e}-06$ & $2.31 \mathrm{e}-05$ & Turquoise \\
\hline Regulation of actin cytoskeleton & 4810 & $1.98 \mathrm{e}-06$ & $2.40 \mathrm{e}-05$ & Turquoise \\
\hline
\end{tabular}


Supplementary Table 2. The results of DO (disease) enrichment analysis of genes in three gene modules

\begin{tabular}{|c|c|c|c|c|}
\hline Gene set & ID & $\mathrm{P}$ & $P_{\text {adjust }}$ & Gene module \\
\hline Li-Fraumeni syndrome & DB_ID:PA165108952 & $1.18 \mathrm{e}-05$ & 0.0018 & Black \\
\hline Translocation, genetic & DB_ID:PA445914 & $4.19 \mathrm{e}-05$ & 0.0032 & Black \\
\hline Myotonic dystrophy & DB_ID:PA445031 & 0.0004 & 0.0087 & Black \\
\hline Raynaud disease & DB_ID:PA445499 & 0.0002 & 0.0087 & Black \\
\hline Mental disorders & DB_ID:PA447208 & 0.0003 & 0.0087 & Black \\
\hline Generalised lentiginosis & DB_ID:PA165108848 & 0.0003 & 0.0087 & Black \\
\hline Anemia & DB_ID:PA443340 & 0.0004 & 0.0087 & Black \\
\hline Nervous system diseases & DB_ID:PA445093 & 0.0010 & 0.0191 & Black \\
\hline Leukemia & DB_ID:PA444750 & 0.0022 & 0.0262 & Black \\
\hline Muscle hypotonia & DB_ID:PA444992 & 0.0021 & 0.0262 & Black \\
\hline Amyotrophic lateral sclerosis & HP:0007354 & $1.45 \mathrm{e}-05$ & 0.0158 & Blue \\
\hline Vascular skin abnormality & HP:0007354 & 0.0011 & 0.1332 & Blue \\
\hline Atrophy/degeneration involving motor neurons & HP:0007373 & 0.0006 & 0.1332 & Blue \\
\hline Abnormality of the dorsal column of the spinal cord & HP:0011397 & 0.0010 & 0.1332 & Blue \\
\hline Decreased sensory nerve conduction velocity & HP:0003448 & 0.0010 & 0.1332 & Blue \\
\hline Abnormal bleeding & HP:0001892 & 0.0009 & 0.1332 & Blue \\
\hline Frontotemporal dementia & HP:0001892 & 0.0010 & 0.1332 & Blue \\
\hline Internal hemorrhage & HP:0011029 & 0.0011 & 0.1332 & Blue \\
\hline Abnormality of blood circulation & HP:0011028 & 0.0011 & 0.1332 & Blue \\
\hline Peripheral neuropathy & HP:0009830 & 0.0019 & 0.1713 & Blue \\
\hline cancer or viral infections & DB_ID:PA128407012 & $4.12 \mathrm{e}-11$ & $3.09 \mathrm{e}-08$ & Turquoise \\
\hline Neoplasms & DB_ID:PA445062 & $6.29 \mathrm{e}-09$ & $2.36 \mathrm{e}-06$ & Turquoise \\
\hline H syndrome & DB_ID:PA162372881 & $1.25 \mathrm{e}-07$ & $3.12 \mathrm{e}-05$ & Turquoise \\
\hline Syndrome & DB_ID:PA445789 & $1.00 \mathrm{e}-06$ & 0.0002 & Turquoise \\
\hline Pigmentation disorders & DB_ID:PA445325 & $1.58 \mathrm{e}-06$ & 0.0002 & Turquoise \\
\hline Mycosis fungoides & DB_ID:PA445010 & $1.01 \mathrm{e}-06$ & 0.0002 & Turquoise \\
\hline Neoplasm of unspecified nature of digestive system & DB_ID:PA165108442 & $3.66 \mathrm{e}-06$ & 0.0003 & Turquoise \\
\hline Lymphoma & DB_ID:PA444840 & $3.18 \mathrm{e}-06$ & 0.0003 & Turquoise \\
\hline Chromosome aberrations & DB_ID:PA443728 & $2.78 \mathrm{e}-06$ & 0.0003 & Turquoise \\
\hline
\end{tabular}


Supplementary Table 3. The results of DO (Drug) enrichment analysis of genes in three gene modules

\begin{tabular}{|c|c|c|c|c|}
\hline Drugs & ID & $\mathrm{P}$ & Padjust & Gene module \\
\hline Daunorubicin & DB_ID:PA449212 & 0.0026 & 0.0222 & Black \\
\hline Divalproex sodium & DB_ID:PA164783479 & 0.0074 & 0.0222 & Black \\
\hline Valproic acid & DB_ID:PA451846 & 0.0074 & 0.0222 & Black \\
\hline Cisplatin & DB_ID:PA449014 & 0.0074 & 0.0222 & Black \\
\hline Phenylephrine & DB_ID:PA449014 & 0.0050 & 0.0222 & Black \\
\hline Doxorubicin & DB_ID:PA449412 & 0.0035 & 0.0222 & Black \\
\hline Quinine & DB_ID:PA451213 & 0.0126 & 0.0284 & Black \\
\hline Cyclosporine & DB_ID:PA449167 & 0.0118 & 0.0284 & Black \\
\hline Glutathione & DB_ID:PA449167 & 0.0181 & 0.0358 & Black \\
\hline Tamoxifen & DB_ID:PA451581 & 0.0199 & 0.0358 & Black \\
\hline Glutathione & DB_ID:PA449780 & $1.13 \mathrm{e}-10$ & $1.89 \mathrm{e}-08$ & Blue \\
\hline Adenosine & DB_ID:PA449780 & $5.28 \mathrm{e}-08$ & $4.41 \mathrm{e}-06$ & Blue \\
\hline Dexamethasone & DB_ID:PA449247 & $9.18 \mathrm{e}-06$ & 0.0005 & Blue \\
\hline Imatinib & DB_ID:PA10804 & $1.24 \mathrm{e}-05$ & 0.0005 & Blue \\
\hline Sirolimus & DB_ID:PA451365 & 0.0001 & 0.0024 & Blue \\
\hline Adalimumab & DB_ID:PA10004 & $8.30 \mathrm{e}-05$ & 0.0024 & Blue \\
\hline Rituximab & DB_ID:PA451261 & 0.0001 & 0.0024 & Blue \\
\hline Progesterone & DB_ID:PA451123 & 0.0002 & 0.0037 & Blue \\
\hline Fluocinolone acetonide & DB_ID:PA164754912 & 0.0002 & 0.0037 & Blue \\
\hline Urokinase & DB_ID:PA164754912 & 0.0003 & 0.0046 & Blue \\
\hline Adenosine & DB_ID:PA448049 & $3.71 \mathrm{e}-15$ & $4.97 \mathrm{e}-13$ & Turquoise \\
\hline Adenosine triphosphate & DB_ID:PA164743471 & $1.87 \mathrm{e}-06$ & 0.0001 & Turquoise \\
\hline Glutathione & DB_ID:PA449780 & $4.10 \mathrm{e}-05$ & 0.0018 & Turquoise \\
\hline Cisplatin & DB_ID:PA449780 & 0.0003 & 0.0100 & Turquoise \\
\hline Insulin recombinant & DB_ID:PA164744571 & 0.0004 & 0.0107 & Turquoise \\
\hline Sorafenib & DB_ID:PA7000 & 0.0006 & 0.0134 & Turquoise \\
\hline Allopurinol & DB_ID:PA448320 & 0.0011 & 0.0211 & Turquoise \\
\hline Mitomycin & DB_ID:PA448320 & 0.0013 & 0.0218 & Turquoise \\
\hline Ganciclovir & DB_ID:PA449733 & 0.0018 & 0.0231 & Turquoise \\
\hline Iodixanol & DB_ID:PA164783998 & 0.0019 & 0.0231 & Turquoise \\
\hline
\end{tabular}


Supplementary Table 4. Prioritization of genes in black module

\begin{tabular}{|c|c|c|}
\hline Gene & Betweenness & Degree \\
\hline EP300 & 171.3333333 & 10 \\
\hline HDAC4 & 110 & 4 \\
\hline NUP153 & 62 & 3 \\
\hline ZNF217 & 47 & 5 \\
\hline MED13 & 32 & 4 \\
\hline AGO1 & 16.33333333 & 5 \\
\hline AGO4 & 16.33333333 & 5 \\
\hline BRCA1 & 12 & 4 \\
\hline SYNJ1 & 8 & 4 \\
\hline TGOLN2 & 8 & 4 \\
\hline PPP3R1 & 6 & 3 \\
\hline NCOA2 & 3 & 4 \\
\hline GAPVD1 & 0 & 3 \\
\hline IGF2R & 0 & 3 \\
\hline KMT2E & 0 & 3 \\
\hline MED13L & 0 & 3 \\
\hline AREL1 & 0 & 2 \\
\hline DICER1 & 0 & 2 \\
\hline HECW2 & 0 & 2 \\
\hline KAT6A & 0 & 2 \\
\hline UBE4A & 0 & 2 \\
\hline APC & 0 & 1 \\
\hline ASH1L & 0 & 1 \\
\hline ATRX & 0 & 1 \\
\hline C11orf30 & 0 & 1 \\
\hline CCNT2 & 0 & 1 \\
\hline CRYBG3 & 0 & 1 \\
\hline EXOC8 & 0 & 1 \\
\hline FOSL2 & 0 & 1 \\
\hline GAB1 & 0 & 1 \\
\hline ITPR2 & 0 & 1 \\
\hline KDM5B & 0 & 1 \\
\hline MYO5A & 0 & 1 \\
\hline NEK6 & 0 & 1 \\
\hline PAG1 & 0 & 1 \\
\hline PIP5K1A & 0 & 1 \\
\hline PPP1R12A & 0 & 1 \\
\hline QKI & 0 & 1 \\
\hline RIC1 & 0 & 1 \\
\hline RNF169 & 0 & 1 \\
\hline SEC24C & 0 & 1 \\
\hline SNTB2 & 0 & 1 \\
\hline SPATA13 & 0 & 1 \\
\hline SSH1 & 0 & 1 \\
\hline TRAPPC10 & 0 & 1 \\
\hline
\end{tabular}




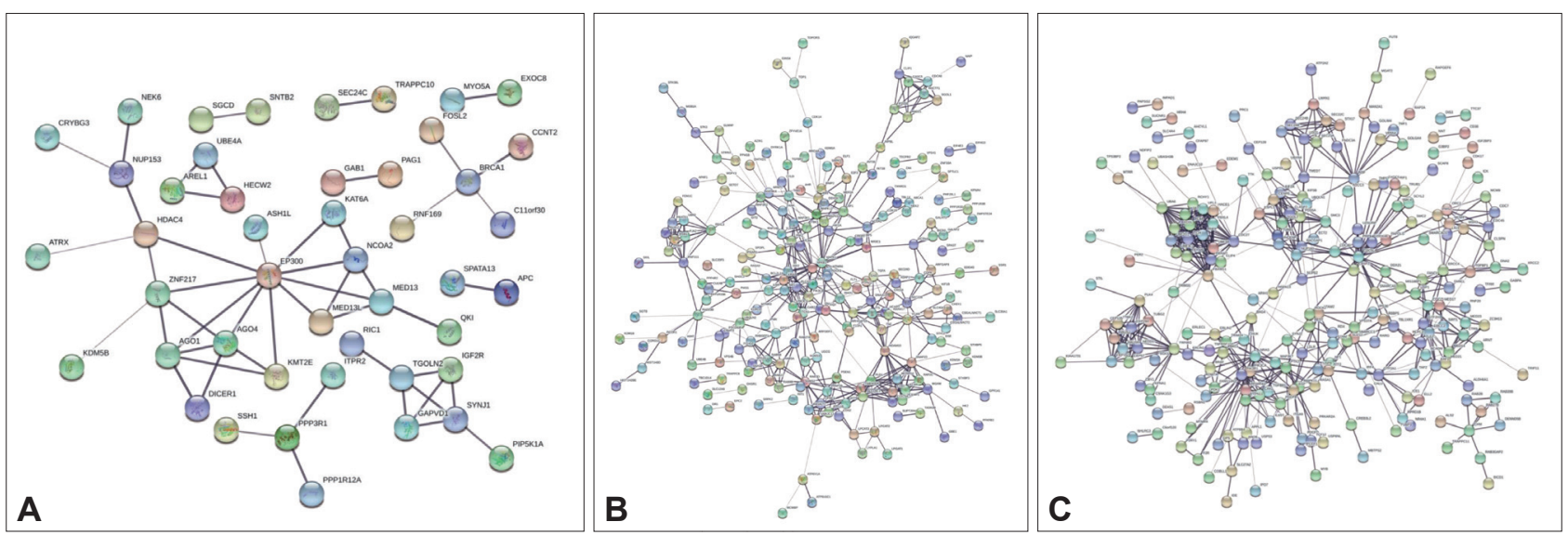

Supplementary Figure 1. Results of PPI network analysis of genes in the three gene modules. A: Black module gene. B: Blue module gene. C: Turquoise module gene. 